Meta

Journal des tradlucteurs

Translators' Journal

\title{
Le traducteur ou par delà l'esclave-maître
}

\section{Michelle Bourjea}

Volume 28, numéro 1, mars 1983

La traduction dans le monde

Translation around the World

URI : https://id.erudit.org/iderudit/002125ar

DOI : https://doi.org/10.7202/002125ar

Aller au sommaire du numéro

Éditeur(s)

Les Presses de l'Université de Montréal

ISSN

0026-0452 (imprimé)

1492-1421 (numérique)

Découvrir la revue

Citer cet article

Bourjea, M. (1983). Le traducteur ou par delà l'esclave-maître. Meta, 28(1),

35-47. https://doi.org/10.7202/002125ar d'utilisation que vous pouvez consulter en ligne.

https://apropos.erudit.org/fr/usagers/politique-dutilisation/ 


\title{
LE TRADUCTEUR OU PAR DELÀ L'ESCLAVE-MAÎTRE
}

\author{
Michelle BouRJeA
}

\begin{abstract}
"Voilà un poème, un livre entier qui ne sont que du noir sur du blanc, le pointillé compact et irrégulier de la page imprimée, et ce qu'on appelle «lettre close»- «attendez un peu», dit le traducteur, et voici que sous sa baguette magique ce qui n'était qu'une triste et grise matière imprimée, illisible imprononçable... devient une parole vivante, une pensée articulée...»

VALÉRY LARBAUD, Sous l'invocation de Saint-Jérôme.
\end{abstract}

Un coup de foudre.

C'est comme ça qu'on devient traducteur. Sur un coup de foudre. «Uma idéia toda azul.» «Une idée couleur d'azur.»

C'est comme ça qu'on se trouve, sans l'avoir vraiment voulu, confronté aux réalités de la traduction. C'est comme ça que, jouant davantage d'un empirisme tout intuitif que d'une véritable expérience professionnelle, on en vient à vérifier le bien-fondé de ses options linguistiques et littéraires et à justifier la pertinence d'une méthode choisie sous le coup de l'inspiration.

Ce travail montre comment furent dépistés et déjoués les pièges inhérents à la pratique de la traduction et comment, à partir d'un besoin de réflexion personnelle et d'un souci d'information théorique, on a été conduit à mieux comprendre le rôle du traducteur et à élaborer une politique/philosophie du traduire moderne. On trouvera donc ici tout autant le cheminement pratique que la démarche intellectuelle qui ont simultanément présidé à la traduction de «UMA IDEIA TODA AZUL» de Marina Colasanti.

«Traduire c'est la manière la plus attentive de lire ».

J. SALAS

Le premier temps d'une traduction est à l'évidence une bonne lecture du texte qui aura été choisi sur un coup de foudre, par une entrée en sympathie immédiate avec l'auteur. Ce n'est pas par hasard que saint Jérôme a traduit la Bible de l'hébreu ni que Baudelaire a traduit Edgard Poe.

De nombreuses lectures approfondies permettent une approche scientifique, basée sur l'observation et l'analyse du matériau linguistique, visant à un rigoureux décodage et évitant les grossières erreurs de langue. Mais une telle lecture est insuffisante et doit se doubler d'une approche plus humaine, esthétique et affective. Se mettant au diapason du texte, le traducteur le pénètre autant qu'il 
s'en laisse pénétrer, établit avec lui un rapport de complicité sans lequel il paraît peu probable d'en cerner justement les caractéristiques naturelles, d'accéder à son unicité et à son tempo, à son dit comme à son non-dit. Ainsi seulement, le traducteur parvient à une telle adhésion au texte qu'il retrouve «l'esprit même dans lequel il a été composé», suivant la fameuse prière à saint Jérôme, patron des traducteurs, reconstituée par Valéry Larbaud.

C'est par une mise à l'écoute de ce type que le traducteur de ces contes en a déterminé les caractéristiques suivantes, afin d'adopter des principes de traduction appropriés :

- Spécificité brésilienne peu ou pas marquée. «C'est le seul de mes livres, dit Marina, qui pourrait être traduit.» Serait-ce de par sa filiation à la littérature populaire et enfantine ou parce qu'il travaille sur des universaux aisément transmissibles?

- Concision et force de la narration.

- Simplicité de la forme touchant à la stylisation alliée à une saisissante singularité de l'expression.

Il en ressort que compte tenu de la facilité de décodage de l'ensemble de ces contes, les problèmes majeurs présentés par leur traduction ne se situent ni dans la recherche du vocabulaire, ni dans la complexité des structures, ni dans l'hermétisme des images. Ils ne sont pas d'ordre linguistique mais esthétique et culturel. Et l'important n'est pas tellement de savoir comment ces contes furent traduits du brésilien en français, mais du français courant en français littéraire. Il s'est donc agi pour le traducteur de rester assez proche du texte brésilien pour lui conserver toute sa saveur et son imprévisibilité, sans toutefois trop dépayser le lecteur français qui aime à retrouver au cours de ses lectures les plaisirs promis par une longue tradition narrative.

Le traducteur a résolu ces problèmes en conciliant puis dépassant deux principes de base :

I - Soumission - calque. Le traducteur adoptant une attitude toute passive se coule modestement dans le texte-source, sans en altérer ni la pureté ni l'originalité.

$I I$ - Subversion - annexion. Le traducteur prenant l'initiative, tire le texte à lui, le francise et justifiant son action par une différence d'expérience historique entre le Brésil et la France, va s'inspirer de la richesse et de la spécificité de l'ancien français pour lui emprunter certaines de ses tournures.

III - Traduction - ré-écriture. Le traducteur se lance dans l'aventure de la création.

\section{SOUMISSION - CALQUE}

\section{Le Lexique}

L'auteur de ces contes ayant volontairement opté pour un vocabulaire extrêmement sobre, porteur de l'idée la plus simple quoique auréolée de connotations diverses, le premier devoir d'un traducteur honnête consiste à le respecter et à ne pas chercher à l'enrichir artificiellement. Ainsi les substantifs «rei», 
«palácio», «floresta», «vento», «lua», «fruta», dans leur contexte, diffusent par leur seule inscription noir sur blanc, toute une atmosphère et condensent une charge poétique symbolique qui les fait se suffire à eux-mêmes.

Cette remarque est particulièrement valable pour les verbes réduits à leur essence. Ainsi en est-il de "ouvir», «ver», "chamar», "chegar», «ir», "descer», «correr», « esperar», «bordar», «querer», «amar»... qui, repris de conte en conte, répètent en écho des gestes immémoriaux. Le traducteur s'attache donc à éviter de les moduler ou de les renforcer en les surchargeant de nuances psychologiques ou physiques. Ainsi conservent-ils intactes leur pureté naturelle et leur force d'évocation initiale.

Dans la même optique, chaque fois que l'origine commune des deux langues le permet, la traduction se fait au plus près de l'étymologie, ne modifiant qu'imperceptiblement la morphologie même des mots. Ainsi «naquele dia» est tout naturellement «jadis», «morar» devient «demeurer», «roubar» «dérober» et «azul» mute en «azur».

\section{Structure de la phrase}

Ici encore le mot d'ordre est : soumission au texte de départ pour peu que la compréhension ne s'en trouve pas obscurcie ni le message dévoyé. Ainsi, quitte à surprendre, le traducteur suit la syntaxe brésilienne et ce, afin de rendre au maximum les particularités stylistiques de la langue.

\section{- Longueur des phrases}

Le traducteur s'efforce de conserver la brièveté des phrases qui sont généralement ramenées à un minimum grammatical, elliptiques de compléments attendus, dépourvues la plupart du temps de lien logique. En effet l'absence régulière de subordination et de coordination, leur fréquente réduction à "et", soulignent la simple succession des événements dans le temps et contribuent à créer, comme naturellement, des effets de merveilleux. Vouloir en ajouter pour satisfaire à un besoin de logique et de clarté française serait méconnaître ce rôle.

- Syntaxe de la phrase

Ici encore le traducteur se garde de modifier la chaîne linguistique et en respecte les unités évitant de les déplacer intempestivement :

«Mas de borboleta nenhuma»

«Mais de papillon point».

De même, il reprendra les multiples inversions verbe-sujet, soit par simple reproduction quand cela est possible, les soutenant au besoin par un «et»:

«Estremece a água do lago»

«Frémit l'eau du lac»

«Brilhavam os dentes abertos em risadas»

«Et brillaient les dents en éclats de rire»,

soit par un redoublement du sujet mettant ainsi le verbe en relief :

«Dizem os pastores da planície...»

«Et ils disent, les bergers de la plaine...»

"Feriam-se os dedos de Gloxinia..."

«Et ils s'abîmaient les doigts de Gloxinie...» 
Enfin il veille à conserver toute leur densité aux phrases nominales, refusant de les fluidifier par quelque verbe passe-partout.

«Um dia mas nada. Nenhuma pegada, nenhum sinal da sua presença. E silencio nas noites».

«(..) un jour plus rien. De sa présence aucune marque, aucun signe. Et le silence des nuits.»

- Répétitions

Voulues par l'auteur, elles ne sont en aucun cas réduites par le traducteur. Elles sont toujours justifiées. Parfois ces retours d'emplois ont une valeur incantatoire. C'est particulièrement le cas des six «enorme» et des quatre «sete» du conte «Sete anos e mais sete » accumulés dans un même paragraphe. D'autres fois, elles soulignent dans la narration le retour d'actions identiques ou l'exacte réciprocité des sentiments. Le traducteur ne joue donc pas au plus malin en les aménageant, car ce serait méconnaître qu'elles modulent la phrase et participent du rythme intérieur de l'ensemble, à la manière d'un motif musical.

3. Figures de style

C'est dans le même esprit que la traduction mimera, autant que faire se peut, les inventions poétiques au lieu de les banaliser par des redressements syntaxiques et des rectifications lexicales. Ainsi «A noite soprou seu vento» par exemple, demeurera : «La nuit souffla son vent» et non, plus traditionnellement : «Le vent de la nuit souffla», qui ne présente aucun intérêt. En effet bien des trouvailles poétiques brésiliennes peuvent parfaitement rendre en français et un calque fidèle des étrangetés de langue-source peut produire un stimulant plaisir de dépaysement.

Cependant un littéralisme aussi aveugle peut aboutir à un aplatissement du signifiant comme du signifié et contrarier l'évasion vers le merveilleux. On peut même dire qu'un si complet effacement devant une cuvre, une si excessive aliénation, touchent au morbide et font d'un texte vivant un texte mort, le réduisant à un respectable code.

Si le traducteur s'est donné pour premier devoir de se mettre à l'écoute du texte brésilien, il se donne un devoir second, celui de se mettre à l'écoute du lecteur. Il est en effet à se demander si le parti pris adopté de soumission est vraiment pertinent pour la traduction de contes. Surtout si cette traduction s'adresse à des lecteurs français de vieilles traditions et longue mémoire, ceuxci ne risquent-ils pas de se sentir frustrés dans leur attente et leur plaisir?

Le traducteur se donne donc pour tâche de subvertir le texte-source dont il brise la neutralité d'une première approche et entre sans complexe dans sa propre langue, récupérant le texte au profit de sa propre tradition.

\section{SUBVERSION - ANNEXION}

1. Par les effets spécifiques de langue

- Temps

Exception faite pour les quelques réflexions parlées qui sont au passé composé, le choix est délibérément porté sur le passé simple, temps narratif par excellence des récits imaginaires se déroulant dans le passé. Par ses désinences 
savantes et son emploi de plus en plus réservé à l'écrit, il émane de lui une coloration, comme un charme désuet, qui a toujours séduit l'enfant comme l'adulte. Ainsi traduit-on: "Foi isso que aconteceu» par «Ce fut ce qui arriva" et non, comme l'usage courant le demanderait : "C'est ce qui est arrivé».

De même le traducteur use sans répugnance du subjonctif imparfait lorsque les concordances ou le sens le réclament, sans chercher à le remplacer par la tournure infinitive comme le suggère souvent le brésilien.

«Parecia não faltar nada»

«Il semblait que rien n'y manquât »

«Brincaram até o rei adormecer»

«Ils s'amusèrent jusqu'à ce que le roi s'endormît »

L'effet vieilli de ces deux temps crée une atmosphère non de préciosité mais de temps révolu et lointain. Ils sont comme autant de formules magiques, de mots de passe pour l'évasion.

\section{- Prépositions}

Compte tenu non seulement de l'étendue de leur gamme et de la subtilité de leur emploi en français, le traducteur peut se permettre de jouer de leur valeur sémantique et grammaticale pour tirer d'insidieux effets tant esthétiques qu'affectifs.

Ainsi la préposition «de» particulièrement polyvalente prend le pas sur «à», «avec», «par» dans les cas de types suivants :

"Sa majesté continuait de (à) dormir...»

"Elle tressa un filet de (avec) ses cheveux... »

«La muraille errodée $d$ ' (par) un vent incessant...»

De même les prépositions les plus communément employées pour introduire des relations spatiales entre les termes sont évitées au profit de plus rares.

«Elle s'en fut $a u$ (dans le) bois la princesse»

«Une poussière brillante... épandue par (sur) le sol»

«Rien ne servait de courir par (à travers) les jardins»

«...planta l'épine d'ivoire en (dans) son cœur»

- Déterminatifs

Là où le brésilien se contente d'un article défini ou indéfini, allant bien souvent même jusqu'à user de l'article zéro conférant au texte une espèce de neutralité atemporelle, le français préfère un adjectif possessif (ou démonstratif) qui charge l'expression d'émotivité. Celui-ci, resserrant les liens de parenté et établissant plus nettement les relations d'appartenance, fait davantage participer le lecteur à la fiction.

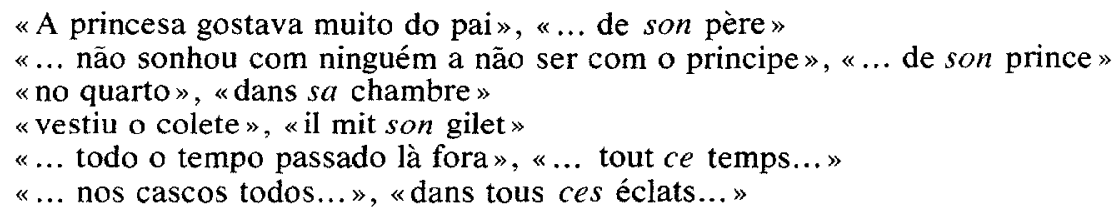

Quant à l'absence d'article, il est généralement stylistique dans ces contes et son effet en est à peu près intraduisible en français. Le traducteur se trouve dans la quasi-obligation d'actualiser et de donner du poids au substantif par l'adjonction d'un article : 
«Kublai-Khan teve desejo de neve...»

«Kublai-Khan eut un désir de neige...»

«Já tinha dono», «elle avait déjà un maitre»

Ainsi la convergence de tous ces choix vers une même rareté-affectivité, contribue à l'homogénéité de la traduction, elle permet d'échapper à la quotidienneté langagière et favorise la création du merveilleux.

\section{Par la musique}

\section{- Vocabulaire}

Au-delà d'une fidélité obtuse au sens et à la morphologie du vocabulaire brésilien, c'est à une recherche de l'exactitude atmosphérique qu'il faut songer, si on veut que le texte ne sonne pas creux en français. Sans vouloir «surtraduire », le choix des mots et des expressions se fait donc sur leur qualité musicale et leurs connotations médiévales.

«Um pouco de mel» — «une once de miel»

«roupa»- «atour»

«Os estudos» - «Ses humanités»

«esquecido do prazo» — «oublieux de l'échéance»

«Gloxinia trabalhava no passar dos anos»

«Gloxinie allait travaillant au fil des ans»

Quelquefois, et curieusement d'ailleurs, l'expression moyenâgeuse recouvre parfaitement l'expression brésilienne, conciliant tout naturellement les deux principes de base du traducteur:

«Um dia indo o rei... visitar a filha...»

«Un jour le roi venant visiter sa fille...»

«Um certo carinho foi se espalhando... »

«Une certaine affection s'en fut se répandant...»

C'est dans cet esprit que le traducteur choisit systématiquement le verbe «être» en remplacement du verbe «aller» et des plus élémentaires verbes de mouvement dans des expressions telles que :

«O rei foi ao quarto da filha» — «Le roi s'en fut...»

«Ela saiu para o bosque...» - « Elle s'en fut au bois»

"Quando o rei veio..." - «Quand le roi s'en vint...»

\section{- Structure de la phrase}

Ici aussi désaliénation par rapport au texte brésilien. Le traducteur n'hésite pas à bousculer la chaîne des mots et des compléments, non pas gratuitement ou par simple esthétisme mais par souci d'adapter la phrase à l'harmonie et au rythme proprement français quand le calque n'est pas pertinent.

«Amanha é o dia» — « Demain le jour sera venu »...

Cette restructuration est particulièrement nécessaire dans les phrases longues :

«Depois ergueu a pipa contra o vento e, abandonando com os pés o alto da muralha da sua fortaleza, deixou-se levar pela corda branca, ultimo Rei Mogol, longe no céu, lá onde ele se tinge de mar. »

«Puis il lança le cerf-volant dans le vent et du haut des remparts de sa forteresse, dans le vide sautant, le dernier Roi Mogol se laissa emporter au loin par la corde blanche, là où le ciel prend la couleur de la mer.» 
Par souci de tirer un parti maximum des tournures moyenâgeuses, certains compléments d'objet ou de lieu précèdent le verbe, comme cela se rencontre souvent en ancien français :

«O rei foi ao quarto da filha...»

"Le roi à la chambre de sa fille s'en fut..."

«Uma borboleta escura pousada num tronco»

«Un papillon couleur de nuit, sur un tronc posé»

«Ela lançou a rede aprisionando o unicôrnio»

"Le filet elle lança et l'unicorne captura »

\section{- Retours sonores}

Le traducteur s'amuse à trouver des rimes internes ou externes toujours faciles, à la manière des comptines enfantines. Cette recherche exige un travail tant rythmique que sonore de la phrase et justifie à elle seule des déplacements syntaxiques importants. Ainsi le traducteur n'hésite pas à rejeter deux verbes à l'imparfait en fin de proposition pour mettre en valeur leur terminaison semblable, à supprimer un pronom sujet pour équilibrer deux propositions juxtaposées et, pourquoi pas, les réduire au même nombre de syllabes, à faire enfin toutes sortes de transpositions grammaticales:

"Usaria o manto, surpreenderia enfim a corte»

«Le manteau elle porterait, la cour enfin étonnerait»

"A primavera desabrochava no manto e a seda desaparecia debaixo das ramagens » «Le printemps s'épanouissait sur le manteau et la soie disparaissait sous les rameaux

«Havia linha, o bordado enriquecia, e Gloxinia trabalhava feliz no passar dos anos» «Abondant était le fil, somptueuse la broderie, heureuse Gloxinie allait travaillant au fil des ans»

«Posta a coroa sobre a almofada, o rei logo levou a mão a corrente.

«La couronne déposée sur le coussin, le roi à la chaîne porta sa main.»

Il est à remarquer cependant que cette seule recherche musicale d'écho et de balancement, si elle justifie de légers écarts sémantiques, s'appuie toujours sur les constructions de type moyenâgeux citées plus haut.

\section{Par les résonances culturelles}

Il s'agit pour le traducteur de donner au texte une coloration culturelle propre à séduire un lecteur français. C'est particulièrement à ce moment-là que le respect du texte d'origine est subverti par un respect plus fort : celui de la tournure née de la tradition proprement nationale, transmise de génération en génération. Ainsi parmi les multiples possibilités de traductions, ce sont toujours les mots, les formules, les tours grammaticaux à la fois les plus rares, les plus oubliés dans leur emploi isolé, mais les plus connus, les plus attendus, les plus assimilés dans leur appartenance à une expression toute faite, véhiculée par tout type de littérature populaire ou popularisée. Jeu de mémoire, jeu de culture. Le traducteur puise dans l'arsenal des contes, comptines, rondes enfantines, chansons populaires, poèmes, fables, dictons et autres mémoires du patrimoine culturel français pour les travailler dans le sens du tableau suivant: 


\begin{tabular}{|c|c|c|}
\hline Expression brésilienne & Expression popularisée & Expression adaptée \\
\hline $\begin{array}{l}\text { - Nem adiantava procurar } \\
\text { no jardim } \\
\text { - Metade mulher, metade cor- } \\
\text { ça, bebendo no regato }\end{array}$ & $\begin{array}{c}F A B L E S \\
\text { - Rien ne sert de courir... } \\
\text { - Un agneau se désaltérait } \\
\text { dans le courant... }\end{array}$ & $\begin{array}{l}\text { - Rien ne servait de parcou- } \\
\text { rir les jardins } \\
\text { - Mi-femme, mi-biche dans } \\
\text { le ruisseau se désaltérant }\end{array}$ \\
\hline $\begin{array}{l}\text { - A cavalo do galho mais } \\
\text { alto da árvore } \\
\text { - Enquanto lançava a agul- } \\
\text { ha... } \\
\text { - Na torre mais alta }\end{array}$ & $\begin{array}{l}\text { CHANSONS } \\
\text { - Sur la plus haute branche, } \\
\text { un rossignol chantait } \\
\text { - Tire, tire l'aiguille ma fille } \\
\text { - Chanson de la plus haute } \\
\text { tour }\end{array}$ & $\begin{array}{l}\text { - A cheval sur la plus haute } \\
\text { branche de l'arbre } \\
\text { - Alors qu'elle tirait l'aiguil- } \\
\text { le... } \\
\text { - Dans la plus haute tour du } \\
\text { château... }\end{array}$ \\
\hline $\begin{array}{l}\text { - Ando sempre en frente } \\
\text { - Os cabelos ainda desfeitos } \\
\text { do sono } \\
\text { - As sementes se aprontam } \\
\text { para a primaveira }\end{array}$ & $\begin{array}{l}\text { TEXTES } \\
\text { - Droit devant soi on ne va } \\
\text { pas bien loin... } \\
\text { - Je suis encore toute dé- } \\
\text { coiffée } \\
\text { - Elle n'en finissait pas de se } \\
\text { préparer à être belle }\end{array}$ & $\begin{array}{l}\text { - Je vais toujours droit de- } \\
\text { vant moi... } \\
\text { - Les cheveux encore tout } \\
\text { décoiffés du sommeil } \\
\text { - Les graines se préparent au } \\
\text { printemps }\end{array}$ \\
\hline $\begin{array}{l}\text { - Corça-mulher só falava } \\
\text { língua da floresta } \\
\text { - Em que todas as pétalas } \\
\text { eram irmăs } \\
\text { - O tempo todo parado }\end{array}$ & $\begin{array}{l}\text { POÈMES } \\
\text { - Le langage des fleurs et des } \\
\text { choses muettes } \\
\text { - Qui lui ressemblait comme } \\
\text { un frère } \\
\text { - Ô temps suspend ton vol... }\end{array}$ & $\begin{array}{l}\text { - La femme-biche ne parlait } \\
\text { que le langage de la forêt } \\
\text { - Les pétales se ressem- } \\
\text { blaient comme des frères } \\
\text { - Tout ce temps suspendu }\end{array}$ \\
\hline
\end{tabular}

Il est cependant nécessaire d'insister sur le fait que le traducteur reste attentif à ne point appliquer artificiellement un patron littéraire ou linguistique tout fait, mais à en jouer seulement sollicité par le texte brésilien, qui doit se prêter à ce jeu sans dévoiement de son contenu.

Ainsi, partant de l'expression brésilienne et traversant l'expression popularisée française, le traducteur adapte l'une à l'autre, conciliant les deux principes de base qu'il s'était proposés. Grâce à ce tissu de réminiscences, le texte gagne en profondeur historique et culturelle. Il s'en trouve orné de quelques fleurons «type français».

À partir de ce face à face concret avec la pratique de la traduction, il s'agit maintenant de s'interroger plus théoriquement sur le statut du traducteur. Tantôt qualifié de traître et de voleur, (pourquoi pas), pour ses initiatives et ses usurpations de texte, tantôt méprisé pour sa fidélité et son renoncement à l'exercice de sa personnalité, celui-ci est depuis longtemps relégué au rang des "domestiques muets et anonymes ${ }^{1}$ de la littérature. Il est donc temps d'analyser par quelles voies insidieuses le traducteur doit s'acheminer, pour mettre fin au discrédit dont il est victime depuis la Renaissance et faire enfin œuvre de créateur.

1. Jean-René Ladmiral, Traduire-Théorème pour la traduction. 
III. TRADUCTION - RÉ-ÉCRITURE «La traduction est l'écriture d'une lecture écri-
ture.»

Meschonnic

Oui, dans un premier temps le travail du traducteur consiste à jouer du double principe de soumission-subversion.

Selon le premier il s'abandonne à un plaisir caméléon, celui de quelqu'un qui prête serment d'allégeance à une langue et une culture étrangères. La seule attitude possible du traducteur est alors la passivité. Usant de la transparence, il ne peut que suivre pas à pas le texte et le mimer. Or, ce n'est qu'exceptionnellement qu'il y a identité sémantique dans le passage d'une langue à l'autre, par simple décalcage. Il s'en suit que le nouveau texte n'est souvent qu'un travestissement maladroit du texte-source.

Selon le second principe le traducteur s'abandonne à un plaisir de trahison par allégeance à ses propres racines, à ses traditions tant langagières que culturelles. Ce plaisir de trahison est essentiel, mais n'est tout à fait légitime que lorsqu'il s'adresse à un lecteur au lourd passé culturel. En effet, si le traducteur veut arriver au terme du plaisir de son lecteur, s'il veut répondre à son attente, il doit céder quasi instinctivement à la fascination de sa propre culture, à son appel implicite, pour faire vibrer en lui les franges spécifiques de la sensibilité nationale. Cependant on peut se demander si, sous couvert de recherche de résonances affectives, le texte n'en devient pas, à sa façon, un autre travestissement, plus séduisant, plus émouvant certes mais tout aussi factice.

Il apparaît donc avec évidence que si le traducteur veut retrouver une dignité et une considération perdue, son souci constant doit être de dépasser ces deux principes de base surmontant par là même la double fatalité de traduire.

Il n'y parviendra que si les affinités qui l'unissent à l'œuvre sont suffisamment profondes et sincères. Car «il s'agit de ne pas se mésallier» ${ }^{2}$. C'est seulement à travers une espèce de relation amoureuse que le traducteur peut espérer faire «la conquête de l'œuvre aimée ${ }^{3}$, participer de son intimité jusqu'à entrer en symbiose avec elle et vivre de sa respiration même.

Ainsi après s'être gentiment reposé à la fois sur le texte brésilien et sur la culture française, le traducteur sort enfin de son assoupissement et adopte une attitude délibérément dynamique. Enfin affranchi, il se fait créateur - s'il le peut. Alors, sous la pression de sa personnalité, le vernis incolore et anesthésiant d'un calque trop scrupuleux craquelle et cèdent les formes sclérosantes de la tradition. Le texte, un moment gelé, réintègre sa pluralité. Le traducteur s'élève au-dessus du chemin étriqué du mot à mot, en secoue les poussières et, s'aventurant dans le débordement sémantique, s'efforce de «traduire non les mots mais l'effet produit ${ }^{4}$ et de capter dans la polysémie retrouvée l'expression susceptible d'établir la correspondance attendue. Car c'est dans le texte qu'il travaille mais surtout dans le contexte. C'est dans la dénotation un peu et dans la

2. Valéry Larbaud, Sous l'invocation de saint Jérôme, p. 93-94.

3. Ibid.

4. Georges Mounin, Linguistique et traduction. 
connotation beaucoup que, de page en page, il traque obstinément la beauté et l'exactitude. Et ce travail n'est jamais ponctuel ni occasionnel, il s'étend à la totalité de l'œuvre et répond à une harmonie interne. C'est pourquoi le traducteur est le grand arrangeur au sens musical du terme. Il est attentif à éliminer fausses notes, ruptures de rythme et autres confusions des registres. Alors, à force d'exhorter et de répudier ses mots, de faire et de défaire ses phrases, de se réjouir et de se repentir, il saisit toutes les nuances et toutes les implications du signifiant comme du signifié et accorde le concert de ses trouvailles pour créer une «langue-système » ${ }^{5}$ forte et cohérente. C'est par cet acte qu'il parvient à un état d'émotion équivalent à celui qui a présidé à la création même de l'œuvre. Offrant toutes les forces vives de sa sensibilité/sensualité, il réalise une traduction qui est «production non reproduction » ${ }^{6}$, et ce faisant il accède à la sphère sacralisée des créateurs.

Alors tout naturellement tombent les travestissements.

Alors sont discréditées les croyances de Humboldt selon lesquelles chaque langue est une vision du monde «irréductible aux autres».

Alors est accomplie la gageure de la belle fidèle ${ }^{7}$ et vaincu le mythe de l'intraduisible, imposant une réflexion sur les vraies fonctions du traducteur dans nos sociétés.

\section{Le traducteur - médiateur}

Si l'on en croit l'étymologie du mot traduire : Traducere : faire passer, tout traducteur est un passeur. Il est le travailleur de l'entre. Entre deux langues, entre deux cultures s'entend. Il est celui qui marque des points de rencontre et tisse de subtils réseaux de connivences entre les cultures. Par son va-et-vient incessant d'une langue-culture à une autre, se soumettant à l'une, subvertissant l'autre et inversement, il est le grand pontife ${ }^{8}$ qui conjure les distances. Sa responsabilité est grande et son action translinguistique n'est dépourvue ni de risques, ni de significations politico-philosophiques. En effet, il en est de l'idéologie politique comme de celle de la traduction : chaque époque, chaque peuple a les traducteurs qu'il mérite. Il en est de colonialistes et de conservateurs. Il en est de progressistes et de libéraux. Il en est de poètes.

C'est un vieux truisme que de remarquer que l'on fait dire ce que l'on veut à un texte mais c'est encore plus évident quand il s'agit d'un texte que l'on traduit. À l'abri des secrets d'une langue étrangère, obnubilé par elle, un texte peut, par incompétence ou volonté d'un traducteur, devenir instrument idéologique, pur monument de morale, vide coquille parée d'esthétisme. Aussi, un traducteur qui veut accomplir au mieux sa fonction se doit avant tout d'être honnête, travaillant au plus près des «structures linguistiques» et des «formes littéraires ${ }^{9}$. Pour cela il lui convient d'adopter une stratégie qui lui évite de tom-

5. Meschonnic, Pour la poétique II, p. 352.

6. Ibid.

7. Par opposition à la Belle infidèle de G. Mounin.

8. Étymologie : faiseur de ponts.

9. Meschonnic, Pour la poétique II, p. 358. 
ber dans les pièges traditionnels inhérents aux traductions. Dans son enthousiasme à gommer les distances.

-- Il fuit ce qu'il est convenu d'appeler «les universaux du langage» qui non seulement affadissent le texte, le lèsent de sa spécificité et de sa part vivante, mais le rendent à peine digne des pires livres de pédagogie de la traduction.

-- Il n'importe pas le texte de langue-source en langue-cible, pratiquant le jeu hypocrite du "comme si». Comme s'il n'y avait qu'un texte directement écrit en langue-cible, commettant ainsi un arbitraire acte d'appropriation.

- Il ne transporte pas enfin le lecteur-amateur-d'exotisme, touriste en chambre, dans un livre-vitrine dont le calque servile et glacé laisse déraper sa sensibilité et glisser son regard de molle curiosité et indifférence.

Dans ces trois cas, il y a bien distances abolies et séparations conjurées par dissolution, assimilation ou réduction à un éphémère produit de consommation, mais toujours artificiellement et toujours au détriment de la langue-source.

Si l'éthique du traducteur est l'approximation au sens brésilien du terme, si sa vocation en notre monde d'extrême médiatisation est d'effacer les distances, elle n'en est pas moins de sauvegarder les différences. Personne en effet ne songe à nier «l'hétérogénéité des mentalités des sociétés, des familles de peuples, des aires et des couches de civilisation», suivant l'expression de Mauss. Ces différences ne doivent être ni rabotées, ni absorbées, ni caricaturées et la fonction du traducteur est de les dynamiser. Chacune d'elles doit devenir pôle d'attraction d'un stimulant échange et engendrer l' «interaction de deux poétiques ». Le traducteur entraîné dans ce mouvement touche à un tel degré de compréhension et de familiarité avec les deux langues qu'il parvient à opérer un «décentrement ${ }^{10}$ et une "ré-énonciation» ${ }^{11}$ du texte-source en une langue qui ne se conforme plus strictement à la langue d'arrivée et qui n'est pas sans affinité avec la langue de départ.

C'est ainsi que l'on peut mieux comprendre ce que peut être une vraie «traduction-texte " en opposition à une «traduction-introduction»" ${ }^{12}$. C'est cet espèce de déplacement linguistique et littéraire si bien rendu par l'anglais «translation " ${ }^{13}$. C'est le produit d'un travail de et dans les langues conservant vivants les liens avec le texte-source et établissant entre les deux langues un rapport dialectique qui aboutit à une féconde rencontre.

Ainsi une réflexion sur la traduction oblige à repenser ce que pourrait être le contact entre les cultures, car la traduction «n'est pas seulement ce qui prend mais ce qui donne ${ }^{14}$. $\mathrm{Ni}$ assisté ni assistant. Ni esclave ni maître. Mais étonnement et fructueux dialogue.

Traduire, éditer des œuvres traduites, ne sont pas des activités philanthropiques qu'on concède aimablement et parcimonieusement à quelques bienheureux auteurs. Ce sont des actes de participation au renouvellement des langues et des

10. Employé par Meschonnic d'après Massignon.

11. Meschonnic, Pour la poétique II, p. 358.

12. Ibid.

13. Ibid.

14. Ibid. 
mentalités. Foin du protectionnisme culturel et linguistique! Il fut un temps dans l'histoire de l'élaboration de notre langue et de notre littérature où l'on s'embarrassait de moins de scrupules et où il suffisait d'une simple «innutrition» pour qu'une œuvre transfuse pour la plus grande exubérance de la culture. Puis vint le temps des geôliers de la langue et du goût, grands arpenteurs, maître peseurs. Et avec eux la déchéance des traducteurs, trafiquants louches. Plus tard, l'engouement pour les voyages et la mode de la littérature comparée réhabilitent quelque peu le traducteur. Aujourd'hui cependant, venant peu à peu à bout de nos nationalismes étanches et timorés, voilà que s'entrebâillent les portes de nos sensibilités et de nos intelligences, laissant venir à nous ces formes et significations venues d'ailleurs. Voilà que quelques maisons d'édition plus audacieuses se lancent dans l'aventure de la production étrangère, ne misant plus seulement sur les «best-sellers» et autres pavés nord-américains.

Ainsi les temps semblent proches du libre-échange culturel où l'on pourra joyeusement se nourrir du grand emprunt. Non point d'un emprunt politicoéconomique plaqué dans le secteur de la rentabilité, mais d'un emprunt lexical, syntaxique et métaphorique. Et l'on s'en nourrira jusqu'à turgescence, fagocytage et dégorgement, jusqu'à rendre insupportables nos plus inviolables idées faites, nos plus innocents poncifs.

L'activité traductrice est à la fois dynamisme, subversion et production. Elle «révèle les possibilités cachées d'une langue ${ }^{15}$, elle est «le possible actif d'une langue ${ }^{16}$. Elle en fait brusquement affleurer les ressources poétiques, émerger toutes les formes latentes, belles dormant au bois de l'inconscient linguistique, en attente d'un poète bien sûr ou d'un traducteur peut-être. Car «le poète est une espèce singulière de traducteur» note Paul Valéry. Le poète comme le traducteur, royalement, insolemment, pose un regard affranchi sur les combinaisons de mots, le corps même des phrases et «traduire entre dans le travail incessant qui change les formes littéraires d'une société ${ }^{17}$. Les surréalistes l'avaient bien compris qui se faisaient un devoir de révolutionner nos images bien filées, nos phrases bien pensantes, eux qui se sont voulus les traducteurs de l'inconscient humain.

Il n'a été question ici bien sûr que de la traduction au sens noble du terme. De celle qui, étrangère à tout désir d'étalage, représentation et autres séductions, loin de se guider dans les sens coincés du dictionnaire, se livre à plaisir aux rythmes d'une autre langue, s'imprègne ${ }^{18}$ de ses visions, en épouse, fascinée, les imprévisibles beautés, en use comme d'un tremplin pour réinventer la langue, lui faire des surprises, l'éblouir de magiques trouvailles.

Ainsi pour n'être pas vraiment poète, le traducteur n'en est pas moins, au même titre que l'acteur ou l'instrumentiste, magistral interprète, génial accoucheur du jamais dit, jamais senti et toujours inouï.

15. C. Robin.

16. Meschonnic, Pour la poétique II.

17. Meschonnic, Pour la poétique $I I$, p. 410

18. Imprégner au sens étymologique de féconder. 
ANNEXI

FIO APÓS FIO

Todas as tardes, na torre mais alta do castelo de vidro, Nemésia e Gloxínia bordavam.

Longo era o manto de seda branca que as duas fadas floresciam e que uma haveria de usar.

Mas Gloxinia, nunca satisfeita com seu trabalho, desmanchava ao fim de cada dia o que tinha feito, para recomeçar no dia seguinte.

Nemésia, gestos seguros, desenhava flores e fôlhas de um jardim em que todas as pétalas eram irmãs, e a cada dia arrematava o ponto mais adiante.

Feriam-se os dedos de Gloxínia de tanto desmanchar. Sujava-se o pano. Os dedos de Nemésia, tranqüilos, brotavam o manto branco.

Faz e desmancha, na cesta de Gloxínia esgotava-se a linha. E ao pegar a última meada, a fada percebeu que não havia avançado um raminho sequer. Caberia à irmã acabar o manto e ficar com ele, sem que ela a nada tivesse direito por seus esforços.

De nada adiantava agora procurar a perfeição. Abandonando por um instante a tentativa de suas pétalas, Gloxínia aproveitou o último fo para bordar sobre a seda, letra por letra, a palavra mágica. Nemésia ainda teve tempo de terminar o ponto e libertar mais uma rosa. Depois transformou-se em aranha.

Gloxinia teria agora tanta linha quanta precisasse.

Paciente, Nemésia teceu o primeiro fio. Que na agulha de Gloxínia revelou-se perfeito, permitindo um bordado certo sem precisar a irmã recorrer à tesoura. Pela primeira vez Gloxínia seguiu sem desmanchar.

Encantou-se com o trabalho. Já não dormia. Colhia o fio da teia mais próxima e logo mergulhava a agulha cantando na cadência dos pontos obedientes. Fio após fio esqueceu-se da irmã. Havia linha, o bordado enriquecia, e Gloxínia trabalhava feliz no passar dos anos.

Chegou o dia do último ponto. Gloxínia acabou uma pétala, arrematou um espinho, e percebeu num sorriso que nada mais havia para bordar: a primavera desabrochava no manto e a seda desaparecia debaixo das ramagens.

Guardada a agulha, Gloxínia levantou-se. Usaria o manto, surpreenderia enfim a corte. Prendeu as fitas largas no pescoço, ajeitou a cauda e virou-se para a porta.

Mas onde estava a porta?

Ao redor de Gloxínia, as teias de Nemésia. Teia encostada em outra teia, que Gloxínia rasgava sem chegar a lugar algum, sòmente a outras e mais teias.

Ao redor da corte, ao redor das salas, ao redor do castelo e dos jardins, lá fora fiava e tecia a paciente Nemésia, esquecida da corte, esquecida da irmã para sempre prisioneira do seu casulo de prata. 